\title{
A37 HLA-DRB1*04 IS A NOVEL FETAL SUSCEPTIBILITY ALLELE IN CONGENITAL HEART BLOCK
}

T Östberg, ${ }^{1}$ S Görgen, ${ }^{1}$ S Salomonsson, ${ }^{1}$ B Ding, ${ }^{2}$ H Eliasson, ${ }^{3}$ Å Elfving, ${ }^{1}$ A Mälarstig, ${ }^{1}$ L Alfredsson, ${ }^{2}$ L Klareskog, ${ }^{1}$ A Hamsten, ${ }^{1}$ T Olsson, ${ }^{4}$ L Padukov, ${ }^{1}$ T Axelsson, ${ }^{5}$ F Gadler, ${ }^{1}$ A Jonzon, ${ }^{6}$ S E Sonesson, ${ }^{3}$ I Kockum, ${ }^{4}$ M Wahren-Herlenius'; 'Swedish Congenital Heart Block Study Group 'Department of Medicine, Karolinska Institutet, Stockholm, Sweden; 'Department of Environmental Medicine, Karolinska Institutet, Stockholm, Sweden; ${ }^{3}$ Department of Women's and Children's Health, Karolinska Institutet, Stockholm, Sweden; ${ }^{4}$ Department of Neuroscience, Karolinska Institutet, Stockholm, Sweden; ${ }^{5}$ Department of Medical Sciences, Uppsala University, Uppsala, Sweden; ${ }^{6}$ Department of Women's and Children's Health, Uppsala University, Uppsala, Sweden

TÖ and SG contributed equally to this work

10.1136/ard.2010.148965.7

Objective Congenital heart block may develop in the fetus of Ro52 autoantibody positive women. The reported recurrence rate for autoantibody associated congenital heart block is however only $10-25 \%$, indicating that other factors than maternal autoantibodies influence the disease development and fetal outcome. The most potent genetic influence on susceptibility to autoimmune diseases is the human leucocyte antigen (HLA) locus, and as there is a strong and well-known HLA-DR*03 allele association with systemic lupus erythematosus, Sjögren's syndrome and production of Ro/SSA autoantibodies, the authors hypothesised that specific alleles of the HLA locus may render the fetus susceptible to congenital heart block induced by maternal autoantibodies.

Methods Genotyping was performed for 561490 singlenucleotide polymorphisms (SNPs) in DNA from Swedish Caucasian families with a Ro/SSA autoantibody positive mother and with at least one case of congenital heart block. Full HLA-A, HLA-C and HLA-DRB1 allele typing was performed by single specific primer PCR in 60 families with complete trios. Swedish Caucasian population-based healthy controls were used in case-control association analysis. Genetic linkage was analysed by transmission disequilibrium test.

Results A case-control analysis between the index cases and population-based controls revealed an association of congenital heart block with six SNPs in the 6p21 major histocompatibility complex (MHC) locus at a genome-wide significance of $\mathrm{p}<5 \times 10^{-8}$ (OR 2.57-3.12). In family-based association analysis of distinct MHC class I and II alleles the authors observed an association of the HLA-DR*04 ( $\mathrm{p}=0.01), \mathrm{DR}^{*} 13(\mathrm{p}=0.02)$ and Cw6 ( $p=0.03$ ) alleles with congenital heart block. HLA-DR*04 was significantly more often transmitted to children who developed congenital heart block, while the opposite was true for HLA-DR*13 and Cw6.

Conclusions This study identifies HLA-DRB1*04 as a novel fetal genetic variant that confers susceptibility to develop congenital heart block in response to exposure to Ro/SSA autoantibodies, while HLA-DR*13 and Cw6 provide resistance. 